\title{
CREATE-NET’s Real-Life Service-Oriented Testbed in Trento
}

\author{
Roberto Grasso, Davide Mandato, Oscar Mayora, Elio Salvadori, Csaba A. Szabó, Luigi Telesca, Hagen Woesner \\ CREATE-NET \\ Trento, Italy \\ \{name.surname\}@create-net.org
}

\begin{abstract}
CREATE-NET's testbed is an open, service-oriented environment, implemented on a real-life city-wide communication infrastructure deployed in the city of Trento. Target user groups and users are integral part of the experimentation and take part in validation of the services implemented in the testbed. It spans the range of the most advanced communications technologies, starting from a wide area DWDM optical backbone, WiMAX \& Wi-Fi wireless distribution/access networks, all the way to sensor networks and technologies for smart spaces. The testbed also serves as a model of a community network, planned for deployment in the Province of Trento.
\end{abstract}

\section{INTRODUCTION}

CREATE-NET's testbed is an open, service-oriented environment, implemented on a real-life city-wide communication infrastructure deployed in the city of Trento. Target user groups and users are integral part of the experimentation and take part in validation of the services implemented in the testbed. It is intended to support live and innovative interaction among individuals, small-to-medium enterprises and public administration entities. Create-Net's testbed is a close relative of the LivingLab type testbeds [1] and is among the first ones of this kind in Europe.

As opposed to most of the service-oriented testbeds, where the network infrastructure itself plays a secondary role, CreateNet's testbed is also technologically strong and innovative. It spans the range of the most advanced communications technologies, starting from a wide area WDM optical backbone, WiMAX \& Wi-Fi wireless distribution/access networks, all the way to sensor networks and technologies for smart spaces, offering:

- $\quad$ real-life broadband pervasive environment to conduct HW/SW research, development and evaluation;

- involvement of target user-groups in the development and evaluation of new services;

- possibility of testing promising technologies with the aim of transferring them into business opportunities and start-ups.

Create-Net's testbed can be part of any Europe-wide distributed experimentation platform through it's connection to the Italian R\&D network (GARR), and the European research infrastructure (GEANT).
The testbed also serves as a model of a community network, planned for deployment in the Province of Trento with the purpose of fostering competition in the telecom market and promoting industry development, to serve the needs of the public sector, and to provide services to the local community.

The paper is organized as follows. In Section II, we introduce the network architecture and present the main technology choices. Section III describes the implemented network infrastructure. In Section IV, innovative extensions of the testbed are presented including the sensor network testbed under implementation, the EPON (Ethernet Passive Optical Networks) access testbed, the service provisioning support and the LivingSpace testbed. Finally, in Section V, services area outlined that are under implementation for two target user groups, university students and SMEs within the framework of the Digital Business Ecosystems.

\section{NETWORK ARCHITECTURE AND TECHNOLOGY SELECTION}

\section{A. $\quad$ Core Network}

Next-Generation-Networks (NGN) applications such us Storage Area Networks (SAN) extension, IP-VPN access, IP$\mathrm{TV}$ and triple-play, mobile backhaul are driving Ethernet growth in a scenario where the final user is already provided with up to $20 \mathrm{Mbps}$ of Internet bandwidth and $100 \mathrm{Mbps}$ is announced. High expectations from carriers are being placed on Ethernet technology to support NGNs as the way forward to support low-cost, high-volume packet networking. Unfortunately Ethernet was originally designed only for localized, single-domain use, and it's application in large-scale access or metro networks requires solution of problems. At carrier grade, Ethernet lacks of interoperability and does not scale well while increasing the number of VLANs. Moreover the fault management and performance monitoring functionalities are weak.

The CREATE-NET testbed aims at addressing these issues (i.e., Ethernet at carrier grade) in a scenario where Ethernet is implemented in all the three segments/layers of the network, interfacing metro distribution optical layer with wireless and optical access network. The following issues are addressed: reliability (network protection within $50 \mathrm{~ms}$ ), hard QoS (guaranteeing an end-to-end service-level agreement), scalability, multi services support (triple play), Operations, Administration, \& Management (OAM) and fault management 
(OAM signaling). Also interoperability and multi vendor support is another topic to which particular care is devoted.

Our approach takes Multi Protocol Label Switching (MPLS) and, in future, Generalized-MPLS from the converged IP/MPLS core out to the edge of the network, most likely with an Ethernet access link to the final user, or a simple aggregation network reaching out to multiple users. Ethernet is encapsulated into the carrier MPLS network (EoMPLS) and networked accordingly. The resilience of the network in case of link failure is provided by 50 millisecond reconfiguration mechanism implemented by the switches. In order to guarantee QoS we have investigated different solutions: preservation of IP-based classification in MPLS headers, identification of flows encrypted within IPSec during WAN handling, traffic shaping in the access to enable grooming diverse applications and VLANs in the WAN. VoIP and Video streaming receive special emphasis because of constrains in terms of delay and jitter. An attractive scenario for the IP QoS implementation is to provide a multiservice environment by connecting large enterprise premises in our testbed core network. Traffic engineering is implemented by using DiffServ in order to fine grain tune many difference levels of QoS.

The presence in the core switches of a physically separate optical layer makes it possible to combine Ethernet (10GbE) with Wavelength-Division-Multiplexing (WDM) solution. So doing it is possible to utilize the advantages of each technology by itself, the benefits of having Ethernet as a service, and the operational/managerial advantages of end-to-end consistency.

The optical layer implements WDM both in Coarse WDM (CWDM) and Dense WDM (DWDM) fashion. CWDM and DWDM have different characteristics. CWDM is based on simpler technology and has restrictions on wavelength count, capacity per wavelength and distance capabilities. However, it is of lower cost than DWDM as it does not require cooled lasers to control wavelength drift. DWDM is based on more advanced technology enabling higher wavelength count, higher capacity per wavelength and longer distances with or without amplifiers. The advantage of having implemented both CWDM and DWDM in CREATE-NET Testbed is twofold. First we can demonstrate a cost-effective CWDM solution that can scale to DWDM if network traffic grows. Second we can dedicate DWDM wavelengths to research activities to experiment new protocols such as Light-trails solutions [2].

\section{B. Distribution/Access Network}

In the access network, a WiMAX-based wireless technology has been selected. The term WiMAX (Worldwide Interoperability for Microwave Access) has become a synonym of the IEEE 802.16 Wireless MAN air interface standard. In its first release, the 802.16 standard addressed applications in licensed bands between 10 to $66 \mathrm{GHz}$ and provided only LineOf-Sight (LOS) application. Subsequent amendments have extended the 802.16 air interface standard to cover Non-LineOf-Sight (NLOS) applications in licensed and unlicensed bands in the $2-11 \mathrm{GHz}$ frequency range (802.16a and $802.16 \mathrm{~d}$ ) as well as mobile applications to enable direct broadband access to mobile devices $(802.16 \mathrm{e})$. Therefore WiMAX systems have a great potential to provide a cost-effective fixed wireless alternative to conventional wireline Digital Subscriber Loop (DSL) and cable in areas where those technologies are readily available.

A wireless MAN based on the WiMAX air interface standard is configured in much the same way as a traditional cellular network with strategically located base stations using a Point-to-MultiPoint (PMP) architecture to deliver services over a radius up to several kilometers depending on frequency, transmit power and receiver sensitivity. The technology was envisioned from the beginning as a means to provide wireless broadband access with performance and services comparable to or better than traditional DSL, Cable or T1/E1 leased line services.

Due to the absence of available $802.16 \mathrm{~d}$ equipment in the deployment phase of the access network, the equipments used in Trento are based on a pre-release of the WiMAX standard, a hybrid between WiMAX and 802.11a working in the unlicensed spectrum $(5.4 \mathrm{GHz})$. In particular, the wireless system is based on a point-to-multipoint topology, with a Base Station (BS) and several Subscriber Stations (SS) installed in several locations around Trento. In some of these locations, a WiFi Access Point has been installed for the final distribution to the final users.

\section{External connections}

Two different connections to the external world have been planned and realized: the first one (Internet) is provided by Alpikom SpA, the main local operator CREATE-NET is collaborating with in some experimental projects on advanced VoIP services. As described in Section III, Alpikom is also hosting one of the optical nodes of the metropolitan network. A connection to the Research and Education network in Italy (GARR), and to GEANT in Europe, has also been realized. This last connection is used to provide remote access to the central facilities to partners involved in research projects.

\section{D. $\quad A A A, I P$ and higher layers}

The testbed implements a solution for user Authentication and Authorization and Accounting (AAA), based on a LDAP+RADIUS server, able to allow secure connection both to partners, users and groups of users to the applications of the testbed.

At application layer, two main applications have been running in CREATE-NET testbed so far: a GRID node and a Service Delivery Platform based on Sun JAIN Open Source Project.

In order to effectively and efficiently manage the service creation and delivery lifecycle across fixed, mobile, and IP networks, telecom providers are increasingly aiming at implementing a converged network architecture called Service Delivery Platform (SDP), which allows applications and services developed for one network type, or services from several networks, to be combined and re-purposed for other networks. SDPs leverage the convergence of IT and telecom technologies, to provide a network-agnostic abstraction layer, to which application developers "write-once-deployeverywhere". 
Session Initiation Protocol (SIP) is an application layer signaling protocol defined by the Internet Engineering Task Force (IETF) for initiating, modifying and terminating communication sessions between endpoints in an IP network. SIP is rapidly becoming the de facto session control protocol that service providers and network operators are using as the foundation for SDP implementations. The convergence of SIP and J2EE technologies is a natural convergence of IT and communication technologies, and when deployed within the SDP, are enabling new IP-based services, such as VoIP.

Standards organizations such as Third Generation Partnership Project (3GPP) have defined industry standard specifications, such as IP Multimedia Subsystem (IMS), for deploying SDPs to extend and evolve existing networks to a next-generation, all-IP network architecture. As high-value IP network services such as instant messaging, web, presence, e-mail are combined with voice and video services, the number of converged IP multimedia applications which can be delivered over IMS networks is expected to grow exponentially.

CREATE-NET has been implementing a SDP based on JAIN JSLEE open source project, on which we are planning to develop innovative services, both for small operators and enterprises, combining instant messaging, web, presence, email, IT applications (CRM-ERP) with voice and video services. The Event technology makes it possible to popular protocol stacks such as SIP to be plugged in as resource adapters offering services to other applications in a distributed scenario. So doing, the presence of a SDP in the CREATENET testbed allows an easy integration and starting-up into the testbed of applications and services developed by external partners.

\section{THE IMPLEMENTED TESTBED}

CREATE-NET testbed combines advanced optical and wireless technologies, deployed in real metro area environment to provide real life users with on-demand, secure and novel services. The testbed is located in the city of Trento including potentially the inhabitants of the town in the experimentation. It supports the technology transfer process of the research center, by providing an infrastructure where local companies or new companies can test and validate their technologies. Fig. 1 illustrates the topology of the testbed and its main architectural parts. Three optical nodes are connected each other by a double optical fiber couples in order to establish a $20 \mathrm{~km}$ ring connecting different premises in Trento. WiMAX and HiperLAN links deploy an access-distribution network based on wireless (802.16 and 802.11 standards) covering potentially all the city of Trento.

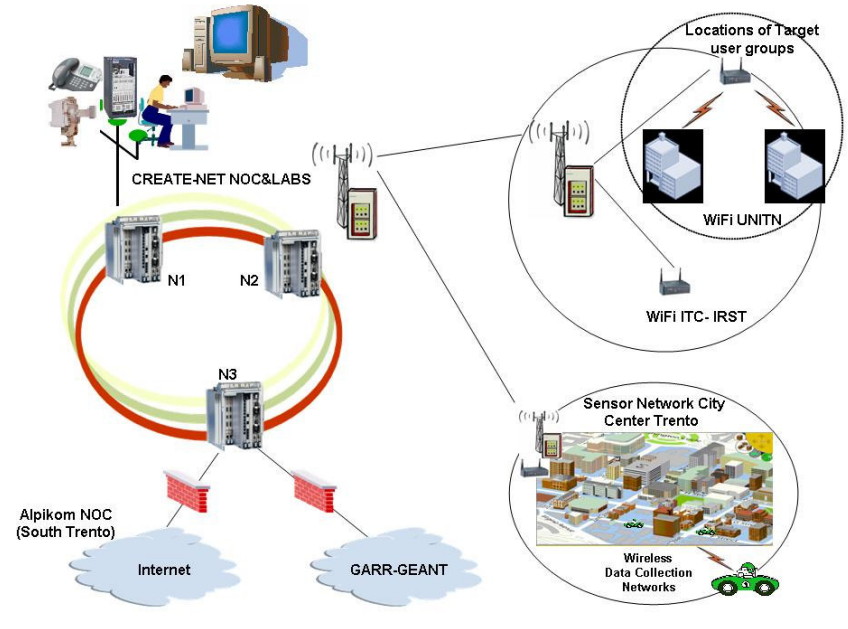

Figure 1. Topology of the testbed

The ring topology of the core network based on WDM separates wavelengths (two 10Gbit Ethernet XGEs) dedicated to operational traffic and wavelengths allocated to experiments

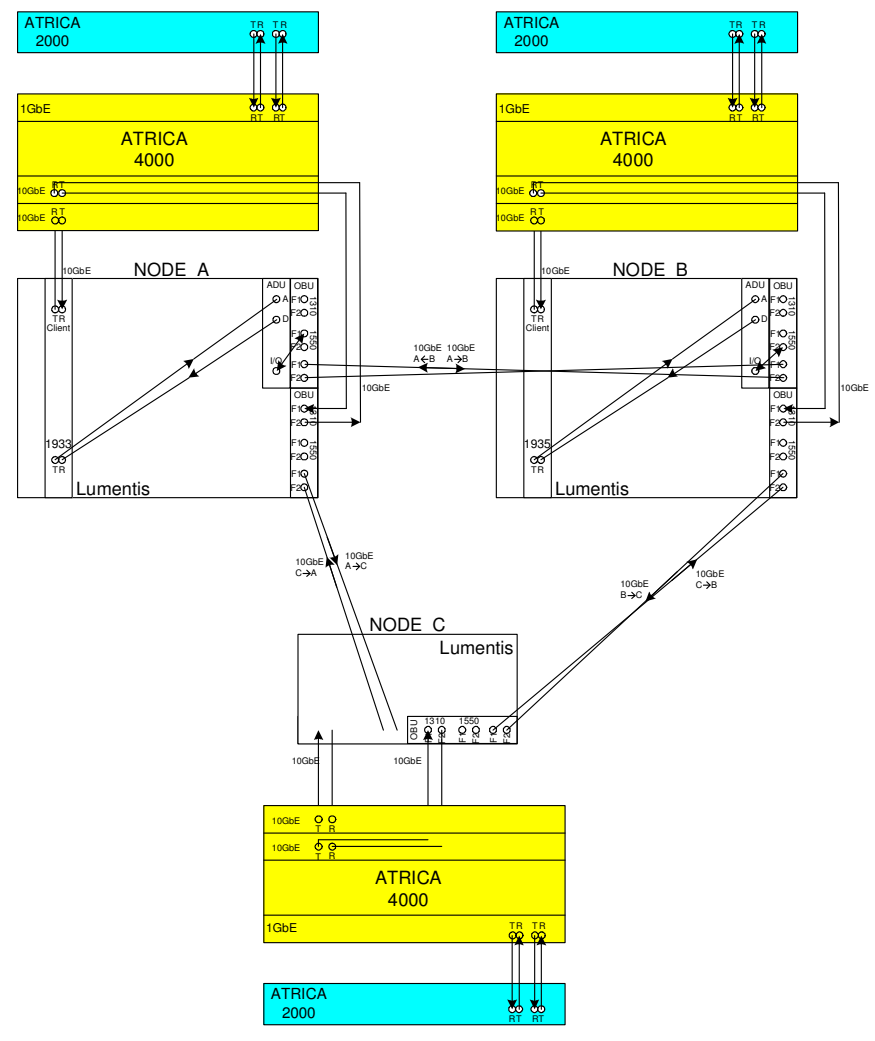

Figure 2. Core nodes interconnection

and new protocols. The optical layer is implemented by using Transmode switches and it is separate from Layer 2 that is based on Atrica A4000 switches (Fig. 2).

Transmode has been the first company able to provide both Coarse WDM (CWDM), Dense WDM (DWDM) and 
SDH/SONET technology on a single modular platform. Transmode optical switches remove the need for complex installation, commissioning and provisioning procedures through automatic client protocol and bit-rate detection.

Atrica A4000 core switches implement the layer 2 of the testbed jointly with A2000 access-aggregation switches. They realize a decoupled approach between the transport layer, which is based on typical layer 2 connection oriented Ethernet services, and the service layer, that comprises MPLS functionalities. MPLS makes it possible to reach fast reroute and up to $50 \mathrm{~ms}$ network recovery. MPLS is managed by ASPEN engine that implements comprehensive management and control of network bandwidth. ASPEN allows enforcing CIR (Committed Information Rate), EIR (Excess Information Rate) and protection to a point-to-point or multipoint-tomultipoint connection ranging up to $1000 \mathrm{Mbps}$. CIR is fully guaranteed from end to end and EIR is especially useful for best-effort data traffic. CIR and EIR per connection provide users with the ability to customize the services for subscribers and offer fast provisioning of services. ASPEN provides APIs that makes it possible to experiment and implement new provisioning systems and algorithms able to engineer GMPLS functionalities. The core equipment implementation is illustrated by the picture in Fig. 3.

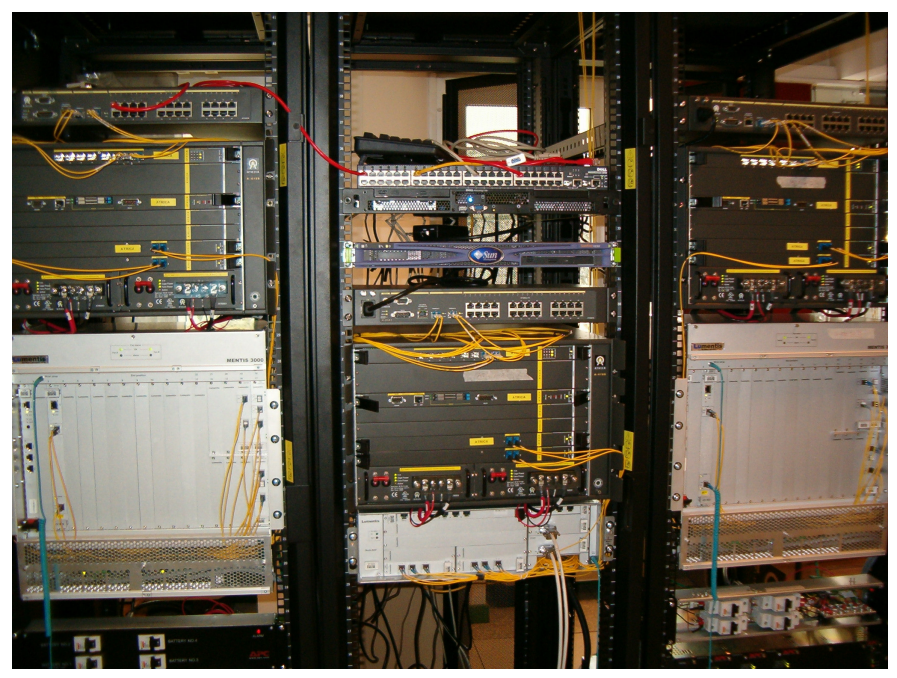

Figure 3. Core equipment in Create-Net central lab

The Alvarion BreezeACCESS VL system uses a prerelease of the WiMAX standard, which is a hybrid between WiMAX and 802.11a. As IEEE 802.11a standard, it works in the $5.4 \mathrm{GHz}$ frequency band and it uses OFDM (Orthogonal Frequency Division Multiplexing) and adaptive modulation. Each channel has the fixed size of $20 \mathrm{MHz}$ while the maximum reachable bit rate is $54 \mathrm{Mbps}$. Furthermore, compared to $802.11 \mathrm{a}$, the system provides a high capacity point-tomultipoint access, Automatic Transmit Power Control (ATCP), Dynamic Frequency Selection (DFS) and a stronger level of security. It also implements QoS mechanisms such as dynamic bandwidth allocation and prioritization. The implemented
MAC layer supports much more users than 802.11a MAC. The duplexing technique implemented in the system is TDD only.

The coverage of the current wireless distribution network is shown in Fig. 4, while an illustration of the outdoor installation is shown in the photo of Fig. 5.

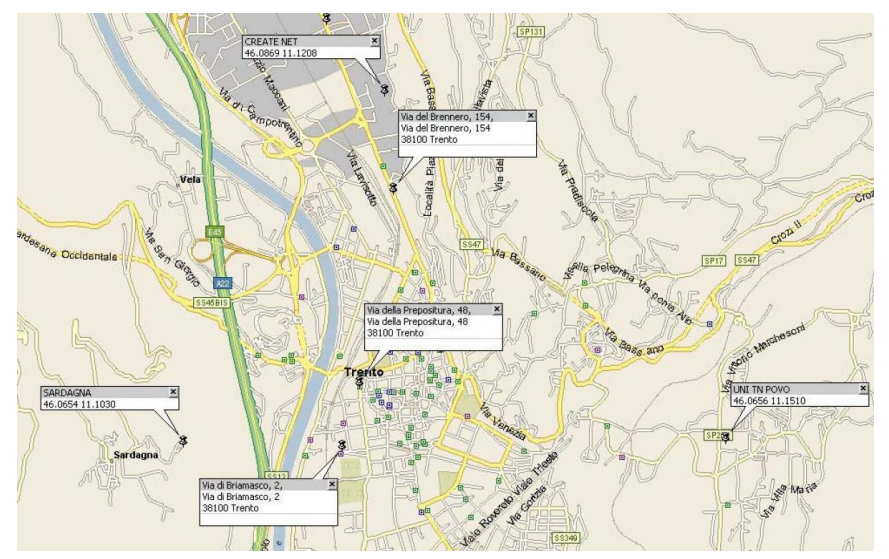

Figure 4. Current pre-WiMAX coverage in Trento

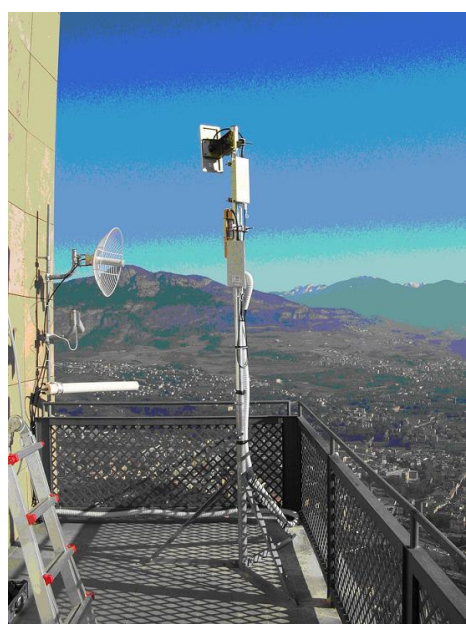

Figure 5. Pre-WiMAX outdoor installation

In summary, Create-Net testbed integrates the following technologies:

- WDM technology for the metro arena both in dense WDM (DWDM) and coarse WDM (CWDM)

- WiMAX and HiperLAN: 802.16 technology seems to be the most promising solution in order to assess last mile connectivity in WLL scenario and rural area to reduce the digital divide. The testbed of Create-Net comprises a multi sector Base Station and a dozen of SUs to cover the area of Trento and to evaluate and to test this technology.

- (G)MPLS aiming at developing effective End-to-End (E2E) network solutions for broadband access guaranteeing acceptable QoS through effective use of network resources with prompt reaction to traffic changes.

- PON IEEE 802.3ah: also known as Ethernet in the first mile, this technology will provide the standard framework 
both for copper and fiber based first mile technologies. We are going to experiment an ePON architecture both in Fiber to Home and Fiber to Base Station fashion. The idea is to implement recent DBA (Dynamic Bandwidth Allocation) algorithms recently developed by Create-Net researchers, and to test it with real users.

- Integration of the University of Trento campus and students hosted by Opera Universitaria for experimentation on $\mathrm{Wi}-\mathrm{Fi}$ and WiMAX in order to have up to 2000 users willing to run and test new services and applications.

- Integration of WiMAX and WiFi networks.

\section{EXTENSIONS OF THE TESTBED INFRASTRUCTURE}

\section{A. Moving to the standard-based WiMAX}

Due to its strong advantage compared to other frequency spectrum both in term of propagation characteristics and to the fact that it is a licensed spectrum, the most common band used by WiMAX technology is $3.5 \mathrm{GHz}$. In Italy this frequency band has not been freed up yet. In order to prepare this change and to evaluate the technology in this frequency range, the Italian Ministry of Telecommunications, in cooperation with the Ministry of Defense, currently using this band, has been setting up a national experimentation on this technology. The experimentation started in autumn 2005 and was coordinated by Fondazione Ugo Bordoni (FUB) [3].

CREATE-NET interest in testing this novel technology mainly comes from its trusted advisory role with the local government (Provincia Autonoma di Trento, PAT). In fact, an ambitious plan to deploy a broadband fixed wireless access network is currently on-going to provide high-speed connections to public administrations and citizen living in remote areas of the province. Although Trento was not included in the Italian areas where this frequency could be used for experimentation, Create-Net decided to participate in a consortium (together with CSP, 2M Telecomunicazioni, Essentia and CRF) in order to perform the experimentation on this technology in Piedmont. To this aim, three different environmental scenarios have been chosen: a rural environment in Canavese, a sub-urban environment in Saluzzo and finally a urban scenario in Turin. All the testbeds have been considering a point-to-multipoint topology, with one Base Station connecting from two up to ten Subscriber Stations. For the experimental setup, we adopted fully IEEE 802.16-2004 compliant equipment operating in the $3.5 \mathrm{GHz}$ licensed band, in particular the solution proposed by Alvarion, called BreezeMAX [4] and the one proposed by Redline Communications solution, named RedMAX [5]. This testbed gives Create-Net the opportunity to perform a wide range of experimental activities on all the different layers of the installed WiMAX infrastructure: further details on the initial measurements campaign can be found in [6][7].

At the time this paper has been submitted it was still not clear what decision the Ministry of Telecommunications will make about the license bid for the $802.16 \mathrm{~d}$ based technology at $3.5 \mathrm{GHz}$ in Italy. In the meantime, a second experimentation phase at national level should be set up by the end of 2006 to test the mobile version of the IEEE 802.16 standard (802.16e). The aim of this experimentation is to test the connectivity to mobile users. Create-Net has already requested authorization to use $3.5 \mathrm{GHz}$ spectrum band in Trento for this second run of national experimentation.

\section{B. $\quad E P O N$}

The success of Ethernet as the ubiquitous technology for packet transport brought up commercial products that range from the original LAN and campus size networks to the backbone of large network providers, thereby replacing SONET/SDH as a physical layer.

While the PHY of Ethernet comes with a huge price advantage, the means to guarantee QoS end-to-end (from and to end users) are not present in Ethernet. In addition, control planes for today's Metro Ethernet products still have multiple standardized interfaces. Current standardization activities for Ethernet control planes are distributed and competing between IETF, ITU, IEEE and the industry-led Metro-Ethernet Forum (MEF). While our metro core testbed uses a proprietary control plane (ATRICA's ASPEN) capable of guaranteeing a committed and excess information rate (CIR/EIR) for VLANs, the extension of these connection-oriented parameters to the end user, i.e. to the desktop or the home entertainment system, is still an open issue.

The IEEE standard 802.3ah, also named Ethernet in the First Mile (EFM) or Ethernet Passive Optical Network (EPON) uses a centralized bandwidth assignment scheme similar to the DOCSIS (Data Over Cable Service Internet Specification) protocol. A single fibre leaves a so-called Optical Line Terminator (OLT) to enter a passive star coupler where the optical signal is split into 16 or 32 tributary fibres that feed Optical Networking Units (ONU). The standard specifies the Multi-Point Control Protocol that is a MAC adapted to the point-to-multipoint nature of PON systems. Note however, that according to IEEE policy any 802 standard only provides a skeleton for bandwidth request and reservation and leaves details of bandwidth allocation algorithms and scheduler implementations to the system manufacturers. Because of this, Dynamic Bandwidth Allocation (DBA) algorithms have been subject to a plethora of publications over the last 5 years.

Providing end-to-end guarantees of bandwidth requires the extension of bandwidth reservation algorithms across the OLT into the metro network. This is the main target of our work on EPON.

This testbed uses FPGA-boards that were developed at COM Denmark. The boards are based on Xilinx Virtex-II chips and equipped with Infineon transceivers providing a downlink transmission rate of $1 \mathrm{Gbit} / \mathrm{s}$. For reasons of timing accuracy at the burst mode receivers the uplink data rate was fixed to 155 Mbit/s. For details of the physical implementation the reader is referred to [8].

The integration of the boards with the Ethernet control plane required the implementation of a DBA algorithm in the OLT board. We chose the simple IPACT algorithm [9] and implemented it inside a soft-core (MicroBlaze) that allows experimentation with DBA algorithms written in $\mathrm{C}$ (instead of 
VHDL). We added support for a serial interface that allows specifying bandwidth parameters according to the MEF recommendations for the UNI [10][11].

This enables the development of a web-services based architecture allowing users to reserve bandwidth for specific purposes, e.g., video conferences, over a web interface. This bandwidth will then be dynamically reserved across the access and metro network. However, several open questions remain, billing of users being one of the more technical, and the reservation towards the "other side" that is users or service providers outside the specific Metro network cloud being a much more challenging conceptual problem.

\section{C. $\quad$ Service Platforms}

Next Generation Networks is a paradigm shift from the classic telco service model of independent, vertically integrated networks to a new architecture that comprises a variety of access networks and has a new horizontal layer or platform that supports service provisioning with important functionalities such as call control, QoS provisioning, media gateways, AAA and the like. This new architecture allows telcos to successfully compete with Internet-based services, and in general, supports the convergence of the Internet, telecommunication and media industries.

Our testbed's strength is its access part as it includes some of the most promising broadband access technologies (WiFi, WiMAX, EPON) that will play an important role in NGNs along with some traditional access solutions offered by telcos and we are going to complete it with service delivery solutions. Our objective is to join a network of interconnected testbeds in Europe so that we can jointly test NGN services, build business relationships with vendors, service providers and system integrators.

This new architecture's key element is SDP or Service Delivery Platform, meaning a set of components that allows an operator to deliver new digital services through a horizontal service network and a multiplicity of access networks. In the narrower (technical) sense SDPs are middleware solutions between the network layer (which is IP) and the applications, the representative example being IMS, the IP Multimedia Subsystem originally developed for $3 \mathrm{G}$ mobile then adopted also by ETSI for its NGN architecture.

We are planning to connect our testbed with a next generation mobile testbed, in particular with specific components of an IMS system recently implemented at the Mobile Innovation Center, BUTE, Budapest, Hungary.

\section{SERVICE-SPECIFIC TESTBEDS}

\section{A. Sensor networks}

Both academic and industry trends in the Pervasive Communications and Computing research field envision a new breed of applications involving mobile users interacting via ever more powerful wireless devices (e.g. cell phones) with a vast number of resource/constrained wireless devices, embedded in the environment. On this breeding ground, a new generation of autonomic context-aware services is expected to appear, featuring the following properties:

- services able to dynamically adapt to ever changing environmental conditions, users ' needs, market trends;

- services capable of coalescing into new, more complex ones in a totally unsupervised fashion;

- services capable of autonomously recovering from faulty situations, without any human intervention

However traditional telecommunication networks, including the Internet and mobile ad-hoc networks, will not be suitable to address the scalability, cost, and decentralized management problems posed by such complex scenarios. To this extent, the identified key research challenges are:

1) identifying new communication and service paradigms;

2) retrofitting already deployed technologies, so as to achieve a seamless architecture that integrates heterogeneous technologies, network domains, and business domains;

3) coordinating these research efforts with those focused on indoors application scenarios (Ambient Intelligence).

To empower this vision, Create-Net is addressing the first aforementioned challenge through the EU-FET Integrated Project BIONETS [12], and the other two challenges through the internally-funded projects. One of these projects, ASSISTMT, has been funded by Provincia Autonoma di Trento (PAT) - Servizio Strade for deploying a system capable of early detecting ice formation on mountain roads. This project, which started in September 2005, deals with the integration of traditional Wireless Sensor Networks (WSN) and disconnected networks (Fig. 6) by means of a Create-Net patent-pending technology. The idea is to offer an ice detection service featuring large geographical coverage (by means of opportunistic routing algorithms) with 24/7 availability (by means of permanently connected WSN confined to strategic areas, each extending over max $2 \mathrm{Km}$ ). Car drivers (currently bus drivers and PAT personnel) are thus able to (a) collect data from isolated sensors and convey it to gateways; and (b) share alerts directly among themselves (e.g. via car-to-car communications). Gateways are meant to (a) control the permanently connected WSN, (b) compute alarms, (c) send alarms directly to PAT personnel via SMS, and (d) relay sensor data via GSM/GPRS connectivity to an operative center, for allowing centralized control.

CREATE-NET is also planning new applications (guidance for car drivers to find free parking places along roads, ensuring public infrastructures safety, providing small businesses with real-time advertisements, enabling efficient garbage collection services).

The idea is to leverage the public transportation system (e.g. buses) as a means to collect a multiplicity of environmental information (including commercial one) from wireless sensors/data sources, and to distribute such information among end-users (either sedentary or on the move ones).

To this extent, the network technologies experimented with the testbed described in this writing will help solving the connectivity constraints posed by rural area scenarios.

For a pretty large class of best-effort, value-added services, though, the aforementioned ASSIST-MT solution can offer a viable solution. A key aspect is in fact that the public 
transportation can effectively act as (i) a vector for physically transporting the information across different geographical locations, and (ii) a means to fuse data and adapt data resolution based on the residual information conveyed by data based on space-time distance from the data origination [13]. A hybrid solution, similar to the one deployed for the ASSISTMT project, is envisioned for solving the service coverage vs. service availability tradeoff. Public transportation will in fact provide the services with large geographical coverage via disconnected networking. But public transportation is necessarily limited to given routes only. In order to guarantee service coverage also in those hot-spot areas adjacent to (but not included in) the public transportation routes, permanently connected WSN are envisioned. Gateways will both coordinate the permanently connected WSNs and act as data collectors of the information gathered by e.g. buses, similarly to what happens in the ASSIST-MT project. The collected data will be delivered to operative centers of institutional parties for coordination/historical archiving purposes. Of course, the role of institutional parties in this model will complement the one of users' communities. For instance, CREATE-NET is developing for the Trento municipality a parking guidance system that will inform drivers in advance about the availability of free parking spaces along roads, possibly before the drivers enter the most congested areas of the city. Of course, the accuracy of the information will be a decreasing function of the distance of the end user from the area of interest [13], whence the information delivered at distant places by e.g. buses will be merely of probabilistic nature, whereas in proximity of the given road it will be detailing the exact number of free parking places. At the same time, the gathered information will be provided to the local authorities for monitoring (aiming at reducing) the parasitic car traffic due to car drivers circling around the most congested city areas looking for a free parking place.

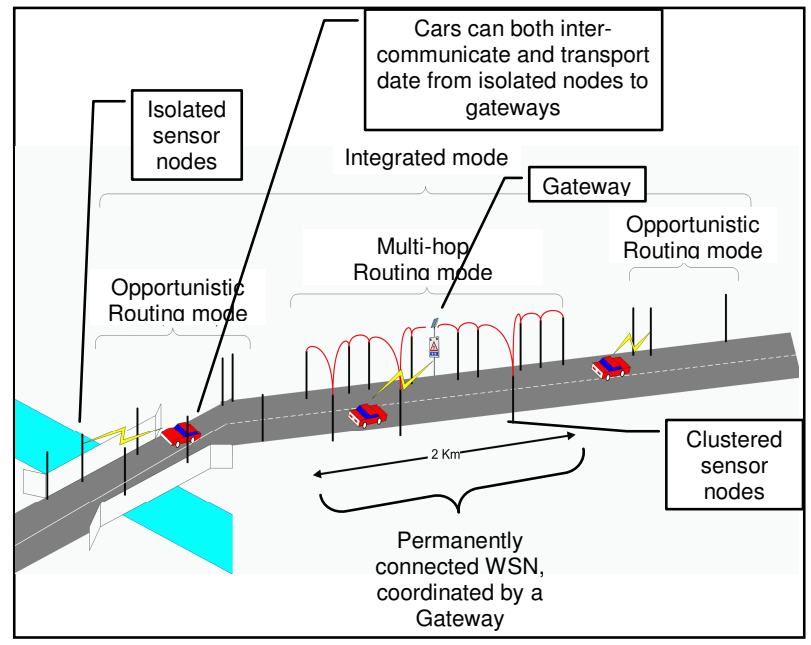

Figure 6. ASSIST-MT hybrid network solution

\section{B. LivingSpace}

One of the main purposes of CREATE-NET testbed is to provide the mechanisms for supporting people in different situations of their daily life. One important piece of people's daily living occurs in indoors environments such as their office, home, etc. The Living Space project represents a complementary part of CREATE-NET testbed, as a container for providing different applications and services on assisted living by combining the indoors intelligent environment with the outdoors smart sensing provided by the testbed. In particular, The Living Space consists in a home-like environment where the main spheres (physical, situational, emotional) in which people conduct their daily life activity occur (see Figure 7).

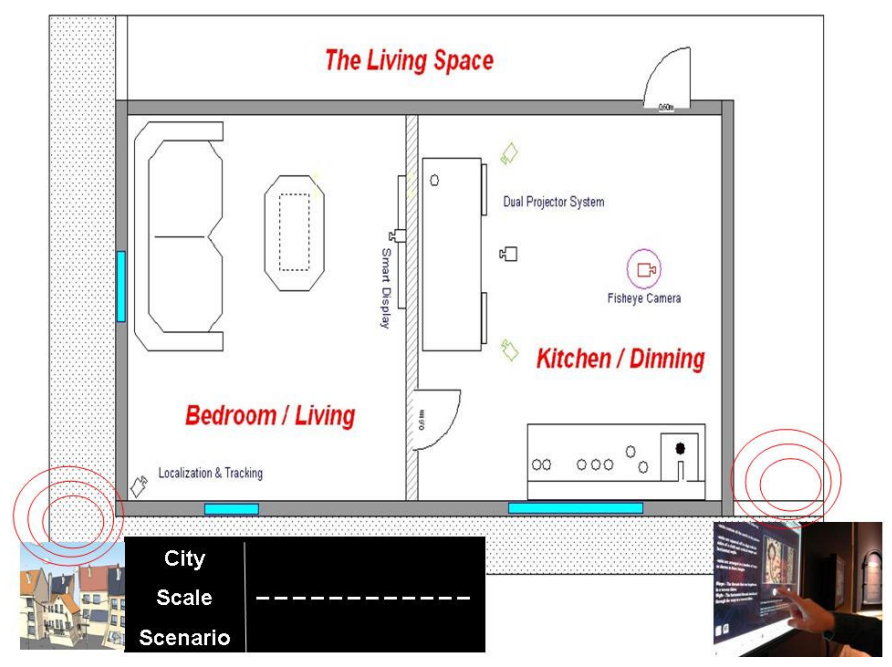

Figure 7. Living Space Smart Environment

The Living Space environment is equipped with different mechanisms for enabling context awareness and proactive interaction with its inhabitants. Examples of ongoing applications in this direction are:

1) Context aware visualization of content through family displays - Ongoing prototype includes a public community display (family environment) that adapts its content accordingly to the person (or persons) detected in its proximity (personalization, profile, etc.) for demonstrating the potential of location and context aware visualization

2) Health emergency detection in indoors environments This prototype describe an application for supporting elderly citizens in emergency situations within home environments. The application is based in a video-surveillance system that enables interactive multi-modal communications between an emergency center and a person in potential health risk [12].

New services envisioned within The Living Space project will explore ambient assisted living technologies based on context, location and intention awareness services, extending the concept of Ambient Intelligence to a broader dimension where pervasiveness and fixed-mobile convergence will coexist. 


\section{Internet connectivity and other services for students}

In the initial phase of the Community Network realized in Trento, the first user group which has been targeted is the student population of the local university (University of Trento). As already described in Section III, three different locations belonging to Opera Universitaria are currently connected through preWiMAX links. The three locations corresponds to student dormitories, each of them able to host from 30 to 100 students.

The initial service currently provided to students is Internet connectivity, but more advanced services are planned in cooperation with the local university.

\section{D. $\quad$ Services for SMEs}

One of the main objectives of Create-Net testbed is to connect different types of target groups in order to experiment innovative technologies and services in real life scenarios with a direct participation of end users in the co-design and testing of the future ICT products and services.

As already mentioned, in the first phase of the testbed's setup we decided to integrate only local students as end users while in the second phase our goal is to involve some other user groups in order to increase the diversity and complexity of the customers base and in order to be challenges by sophisticated application scenarios and business oriented processes.

The local Small and Medium Enterprises (SMEs) and in particular the local software companies have been identified as the second testing group to be engage in the testbed experimentation. This decision has been taken both because of the strategic role that software SMEs play every day in the Provincial innovation process and both for the strong relations they have with the local communities and business end users.

The first step made to engage local Software SMEs, together with their ecosystems, in our experimentation has been to provide them a new software platform developed during an FP6 European project called Digital Business Ecosystem [15]. The comprehensive goal of the DBE has been to provide Europe with a recognized advantage in innovative software application development by its SME industry befitting from the enhanced capabilities of the platform.

The DBE is an open source, internet-based $\mathrm{p} 2 \mathrm{p}$ environment where business applications can be deployed, discovered and consumed and where software companies, even the smallest, can contribute in the creation of business applications. The environment could be used in order to overcome the isolation of SMEs through the creation of ad-hoc and permanent collaborations for strategic production and also as distribution channel.

In this testing scenario Create-Net as Regional Catalyst, will make accessible the testing infrastructure to SMEs willing to cooperate in the creation of a distributed software factory. In this environment all participants could adapt already available software, or develop new ones, and applications on top of the distributed environment while modeling their business model via (Business Modeling Language) BML in a totally controlled environment. BML contains information like: service offered and requested, resources, processes, business model and motivation, policies and agreement, location and event related to business and so on.

Later on we will integrate together with SMEs end users that will test the services developed by the software companies connecting their business with our testbed.

\section{CONCLUSIONS AND FUTURE WORK}

In this paper, we reported on Create-Net's innovative reallife, service-oriented testbed. We described the network architecture, implementation experience, extension of the basic testbed with new technologies and service platform, and the service-specific testbeds connected to it.

Ongoing work includes addition of a standard based NGN service platform and development and deployment of new services for and in cooperation with specific target user groups. The integration of some of the testbeds is matter of further research activities.

\section{REFERENCES}

[1] M. Eriksson, V.-P. Niitamo, S. Kulkki, "State of the art in utilizing Living Labs approach to user-centric ICT innovations - an European approach", Report, Dec 15, 2005, available at www.cdt.ltu.se.

[2] Y. Ye, H. Woesner and I. Chlamtac,"OTDM Light Trail Networks", in Proc. ICTON2005, Barcelona, July 2005

[3] FUB WiMAX experimentation: http://wimax.fub.it

[4] Alvarion: http://www.alvarion.com/

[5] Redline Communications: http://www.redlinecommunications.com

[6] P. Imperatore, E. Salvadori, I. Chlamtac, "Path Loss Measurements at 3.5 GHz: A Trial Test WiMAX Based in Rural Environment”, Accepted for publication, IEEE Tridentcom 2007, May 2007

[7] N. Scalabrino, F. De Pellegrini, I. Chlamtac, et al, "Performance Evaluation of a WiMAX Testbed under VoIP Traffic," Poster paper accepted in ACM WiNTECH 2006, September 2006

[8] M. B. Thomsen, T. S. Lyster et al, "Development platform for Dynamic Bandwidth Allocation schemes in future MPCP enabled Passive Optical Network (EPON)" WSEAS Transactions on Communication, Issue 1, Volume 5, January 2006.

[9] G.Kramer, B.Mukherjee et al., "On supporting differentiated classes of service in EPON-based access network," OSA Journal of Optical Networking, vol. 1, no. 8/9, pp. 280-298, 2002.

[10] Ralph Santitoro ,"Bandwidth Profiles for Ethernet Services", White paper, MetroEthernetForum, www.metroethernetforum.org

[11] Ethernet Services Attributes Phase I, MEF Technical Specifcation 10, www. metroethernetforum.org

[12] FP6-027748, BIOlogically inspired NETwork and Services, www.bionets.eu

[13] I. Carreras, I. Chlamtac, F. De Pellegrini and D. Miorandi, "BIONETS: Bio-Inspired Networking for Pervasive Communication Environments", IEEE Transactions on Vehicular Technology, 2006.

[14] De Natale F., Mayora-Ibarra O. and Prisciandaro L., "Interactive Home Assistant for Supporting Elderly Citizens in Emergency Situations," Proceedings of EUSAI Workshop on AmI for Wellbeing@Home, Eindhoven, Netherlands, November 2004.

[15] Digital Business Ecosystems: http://www.digital-ecosystem.org 